\title{
DNA damage response and disorders with hematology, oncology and immunology
}

\author{
Shuki Mizutani ${ }^{1,2}$
}

Received: 4 July 2017 / Accepted: 5 July 2017 / Published online: 11 July 2017

(C) The Japanese Society of Hematology 2017

One of the recent topics in medical science is provided by the research on congenital human disorders with genetic defects in DNA repair and damage response pathway.

The conditions such as disorders of impaired nucleotide excision repair, DNA double- or single-strand break repair, or compromised DNA damage-induced signal transduction essentially reinforce the importance of genomic stability pathways for health and development in humans. The genomic stability pathways are also becoming more important by the potential applications to therapeutic strategy in various cancers.

Genomic DNA is constantly under threat owing to endogenous or exogenous stimuli such as replication errors, metabolic by-products like reactive oxygen species, or exposure to environmental mutagens like air pollutants et.al. or anti-tumor agents in case of cancer treatment. Other than these stimuli, $\mathrm{V}(\mathrm{D}) \mathrm{J}$ recombination process during normal lymphocyte development involves a signaling pathway for proper DNA double-strand breaks (DSB) repair.

It is essential for the maintenance of genomic stability to prevent the replication of DNA errors. Eukaryotic cells have evolved a machinery for DNA damage response (DDR), constituted by a network of biochemical pathways that detect and repair the DNA lesions, associated with the transient cell cycle arrest or apoptosis, leading to the prevention of the expansion of potential cancer cells.

Shuki Mizutani

smizutani.ped@tmd.ac.jp

1 Professor Emeritus, Tokyo Medical and Dental University, Tokyo, Japan

2 Kawasaki North Center for Childhood Developmental Disorder, Kawasaki, Japan
The association of DDR defects with cancer is best attested by the existence of cancer-prone patients carrying germ line mutations in the genes involved in DDR. Notably, the DDR triggers other molecular events that regulate stem cell homeostasis and aging, and defects in DDR can markedly impact these cellular properties.

Dr. D. Delia will present a latest overview on the heart of the DDR, which is rapidly activated by different DNA breaks and phosphorylates a multitude of substrates, allowing them to jointly orchestrate DNA repair and cell recovery or death. The essential role of DDR in HSC maintenance and protection against leukemogenesis, and how acquired DDR dysfunctions or pharmacological agents that block this pathway can be effectively exploited for the treatment of various hematopoietic malignancies will be presented and discussed.

Patients with Fanconi anemia (FA) are impaired in a pathway that repairs interstrand crosslinks (ICLs). This defect in ICLs is considered the major contributor to the disease state. Notably all FA genes identified so far have been demonstrated to have a role in ICL repair. The defect in DNA repair, which can be a cause of genetic instability, explains the cancer predisposition and ICL sensitivity in FA patients. Furthermore, experimental models have revealed that the bone marrow failure associated with the disease is caused by a response to genetic instability. Drs. Ronald S. Cheung and T. Taniguchi will discuss recent investigations into the molecular pathogenesis of FA within a framework comprised of three parts: genes, modifiers and drivers. The discovery of new FA genes enables a more complete understanding of the canonical FA pathway, leading to potentially targetable biochemistry and enzymology.

Dr. T. Morio focused on Primary immunodeficiency (PID). V(D)J (variable: V, diversity: $\mathrm{D}$, and joining: $\mathrm{J})$ recombination in antigen receptor genes is a DNA 
rearrangement process that occurs at Immunoglobulin heavy chain $(\operatorname{IgH})$ loci, Immunoglobulin light chain loci (IgK and $\operatorname{Ig} \lambda$ ), and at $\mathrm{T}$ cell receptor loci (TCR $\alpha, \beta, \gamma, \delta$ ), which is critical in development of $\mathrm{B}$ and $\mathrm{T}$ lymphocytes. Immunoglobulin class switch recombination (CSR) at $\operatorname{IgH}$ constant regions results in the production of $\operatorname{IgG}, \operatorname{IgA}$, or IgE. RAG1/2 lymphoid specific factors initiate DSB formation in $\mathrm{V}(\mathrm{D}) \mathrm{J}$ recombination; and activation-induced cytidine deaminase (AID) triggers DSB formation in CSR response. Inability to persecute the programmed DDR and repair results in the developmental disorder in T, B cells, that is, primary immunodeficiency (PID). These PID patients often show one or more of phenotypes that include radiosensitivity, predisposition to malignancy, developmental delay, or neurological deficits such as microcephaly, which has been called XCIND [1].

These disorders provide us with an insight into physiological importance and function of each molecule in health and disease. The review will mainly focus on phenotypes of PID due to impaired DDR including newly discovered disorders.

Dr. M. Takagi provides a review on the series of congenital immunological and hematological disorders caused by germline mutation of DDR-associated genes, with an attention to cancer-susceptible syndromes. The review also demonstrates somatic mutations of DDR-associated genes reported in various tumors, including hematological malignancies. Therapeutic approaches that target the DDR and DNA repair are now being investigated. Thus understanding the mechanism(s) underlying DDR and DNA repair will increase our knowledge of cancer etiology and facilitate development of cancer therapies.

In this review series, I picked up only some of the key disorders described within the last decade, giving a flavor of the progress in this important area of the DDR and DNA repair. I hope by this opportunity readers to extend scientific interest in this exciting field of congenital human disorders with the failure of DDR that has not been covered here.

I am convinced that with the promising exciting potential of next generation sequencing, we will uncover more undiagnosed disorders, and then provide extensive understanding of DDR in normal and pathologic conditions of human development.

\section{Reference}

1. Mizutani S, Takagi M. XCIND as a genetic disease of X-irradiation hypersensitivity and cancer susceptibility. Int J Hematol. 2013;97(1):37-42. 